\title{
"String of pearls sign" on FDG PET imaging in two patients with PUO of diverse etiologies and rare associations
}

\author{
Shrinivas Yuvan", Shanmuga Sundaram Palaniswamy ${ }^{2}$ and Padma Subramanyam ${ }^{2^{*}}$ (D)
}

\begin{abstract}
Background: Pyrexia of unknown origin (PUO) may be related to several pathologies that need to be identified for proper treatment. PET is found to have highest diagnostic yield in identifying various causes of PUO. The aim of this study is to highlight and justify the use of ${ }^{18} \mathrm{~F} \mathrm{FDG} \mathrm{PET} \mathrm{(Fluorine} \mathrm{Fluorodeoxyglucose} \mathrm{Positron} \mathrm{Emission}$ Tomography) imaging as a whole body screening tool in two unique cases of febrile illness with lymphadenopathy but with diverse etiologies based on PET-guided biopsy. The unique arrangement of PET positive nodal disease as a "string of pearls sign" helps in easy identification of nodal disease.

Case presentation: The first case presented with fever and headache (past history of meningitis), high ferritin level, leukopenia, anemia, and raised inflammatory markers. CSF cell count was high, with mildly elevated protein and low glucose levels. PET positive nodes were biopsied; Kikuchi Fujimoto disease was confirmed with unexpected FDG avid pachymeningeal enhancements on PETMR indicating associated (active) meningitis which would have been missed if $C T$ or MR was done as a standalone imaging. Lumbar puncture confirmed aseptic meningitis. The patient was treated with anti-inflammatory drugs, inj. methylprednisolone, and immunoglobulin together with hydroxychloroquine. The patient improved on follow-up. The second case presented with low-grade fever, pruritis, and nodular swellings in extremities, anemia, and pancytopenia. Based on PETCT findings, biopsy was attempted from FDG avid axillary nodes. Mantle cell lymphoma with rare nodular involvement of cutaneous and subcutaneous tissue was confirmed. Chemotherapy and tyrosine kinase inhibitor therapy was initiated, and the patient is doing well clinically.

Conclusion: The clinical impact of PET is twofold in both cases. It has accurately identified the nodal involvement even those subcentimetric in size by exhibiting a unique sign on PET resembling a "string of pearls" in the neck and chest with unrelated diverse etiologies. Secondly, additional findings of meningitis in the first case and cutaneous/subcutaneous nodular lymphomatous deposits in the second patient were possible only with whole body FDG PETMR/CT imaging. The rate of additional disease detection by PET is found to be greater than other conventional imaging modalities due to the functional basis of investigation.
\end{abstract}

Keywords: Fever of unknown origin, Whole body FDG PETMR, PETCT, Lymphadenitis, Aseptic meningitis, NHL, Cutaneous nodules

\footnotetext{
* Correspondence: drpadmasundaram@gmail.com

${ }^{2}$ Department of Nuclear Medicine \& Molecular Imaging, Amrita Institute of

Medical Sciences, Ponekkara Post, Cochin, Kerala 682041, India

Full list of author information is available at the end of the article
}

\section{Springer Open}

(c) The Author(s). 2021 Open Access This article is licensed under a Creative Commons Attribution 4.0 International License, which permits use, sharing, adaptation, distribution and reproduction in any medium or format, as long as you give appropriate credit to the original author(s) and the source, provide a link to the Creative Commons licence, and indicate if changes were made. The images or other third party material in this article are included in the article's Creative Commons licence, unless indicated otherwise in a credit line to the material. If material is not included in the article's Creative Commons licence and your intended use is not permitted by statutory regulation or exceeds the permitted use, you will need to obtain permission directly from the copyright holder. To view a copy of this licence, visit http://creativecommons.org/licenses/by/4.0/. 


\section{Background}

Pyrexia of unknown origin (PUO) was first defined by Petersdorf and Beeson in 1961 as recurrent fever of 38.3 ${ }^{\circ} \mathrm{C}$ or higher, lasting 3 weeks or longer, and undiagnosed after 1 week of hospital evaluation [1]. Many cases of febrile illness need a higher line of management than treatment with non-steroidal analgesics and broad-spectrum antibiotics. Non-responders need specialized investigation like FDG PET to identify the site of occult infection or malignancies manifesting as PUO [2].

PUO may be categorized under the following subgroups [3]
a) Infection (20\%-40\%),
b) Malignancy $(20 \%-30 \%)$,
c) Non-infectious inflammatory diseases (10-30\%),
d) Miscellaneous (10-20\%), and
e) Undiagnosed (up to 50\%).

Common infections can be evaluated and diagnosed by simple investigations like complete blood count or urine culture. For others like chronic tonsillitis, osteomyelitis, pyelonephritis, and thyroiditis, specific symptoms and signs elicited during clinical evaluation will be helpful. Malignancies like lymphoma and lung cancers; non-infectious inflammatory diseases like connective disease and vasculitis; and granulomatous diseases like tuberculosis and sarcoidosis will need higher investigations like endoscopy, biopsy from suspicious sites, highresolution CT, MRI, and nuclear medicine techniques for clinching the diagnosis [3].

The number of patients with non-infectious inflammatory diseases and undiagnosed fevers are more common in developed countries, while higher proportion of PUO due to infection and neoplasm are seen in underdeveloped countries. It has been long recognized that ${ }^{18} \mathrm{~F}$ FDG accumulates not only in malignant tissues but also at sites of infection and inflammation and in organs affected by autoimmune diseases. FDG imaging can detect acute and chronic inflammatory processes, infectious sites, and a wide variety of malignancies, all of which exhibit increased glucose metabolism and are potential causes of PUO. FDG PET can identify early metabolic changes in cells and tissues by a process called metabolic trapping during glycolysis. These changes take place ahead of the morphological changes that are delineable by other imaging modalities. PET also risk stratifies and documents the exact disease burden which may not be apparent on regional $\mathrm{CT} / \mathrm{MR}$ with no additional radiation burden. Metabolically active normal-sized diseased lymph nodes are abnormal and can be demonstrated only on FDG PET, unlike CT or MR where size is an important criterion. FDG is a simple glucose molecule that is metabolically trapped in infective, inflammatory, or malignant cells and does not undergo further metabolism through a glycolytic pathway, GLUT (glucose uptake transport) receptors [4]. FDG PET thereby helps in identifying the extent of disease and accurate site for biopsy and facilitates early diagnosis and treatment [4]. Node displaying highest SUV max with easy approachability for biopsy is found to have the highest diagnostic yield that can lead to the right management [4].

\section{Case presentation \\ Case 1}

A 48-year-old lady with recurrent meningitis in the past presented with low-grade fever and headache for 4 weeks duration. There was no associated sore throat, chills, rigors, or any other organ-specific symptoms like burning during micturition. On examination, the patient had fever of 100 degree Fahrenheit and pallor with no palpable nodes or any other positive meningeal signs. Investigations revealed high ferritin $(5269 \mathrm{ng} / \mathrm{ml}$, normal range 15-150), leukopenia, anemia, and elevated ESR ( $45 \mathrm{~mm}$ at 1st hour), and C-reactive protein was $47 \mathrm{mg} /$ dl. Serum, blood, and viral markers for infections, connective tissue disorders, and tumor markers were negative. No vegetations were noted on echocardiogram. Bone marrow aspiration showed dilute marrow. Marrow biopsy revealed micronormoblastic erythropoiesis with mild dyserythropoiesis, negative for acid-fast bacilli. CSF cell count was high $\left(1000\right.$ cells $\left./ \mathrm{mm}^{3}\right)$, mildly elevated protein with low glucose levels. Whole body ${ }^{18}$ F FDG positron emission tomography-magnetic resonance imaging (PETMR) revealed FDG avid bilateral cervical, supraclavicular, axillary, and deep pectoral nodes arranged in a string of pearls pattern on both sides of neck and chest (Fig. 1A). Periportal, portocaval and inguinal nodes were also involved and displayed FDG avidity. Left axillary node biopsy confirmed histiocytic necrotizing lymphadenitis, also known as KFD. FDG PETMR transaxial images (Fig. 2) show abnormal focal increased FDG uptake in MR detected diffuse enhancement and thickening of pachymeninges bilaterally (left > right) with a maximum thickness of $5 \mathrm{~mm}$ on the left side and $3 \mathrm{~mm}$ on the right side, suggestive of meningitis. Lumbar puncture confirmed aseptic meningitis. Fever was treated with non-steroidal anti-inflammatory drugs, along with oseltamivir, ceftriaxone, and doxycycline. The patient was also treated with inj. methylprednisolone 48 milligram (mg)/day and intravenous immunoglobulin (2 $\mathrm{g} / \mathrm{kg}$ in total) together with hydroxychloroquine $200 \mathrm{mg}$ twice daily. The patient improved after $3-4$ weeks and is doing well on follow-up.

\section{Case II}

A 61-year-old male with low-grade fever for 5 weeks, generalized pruritis, and history of a few nodular 


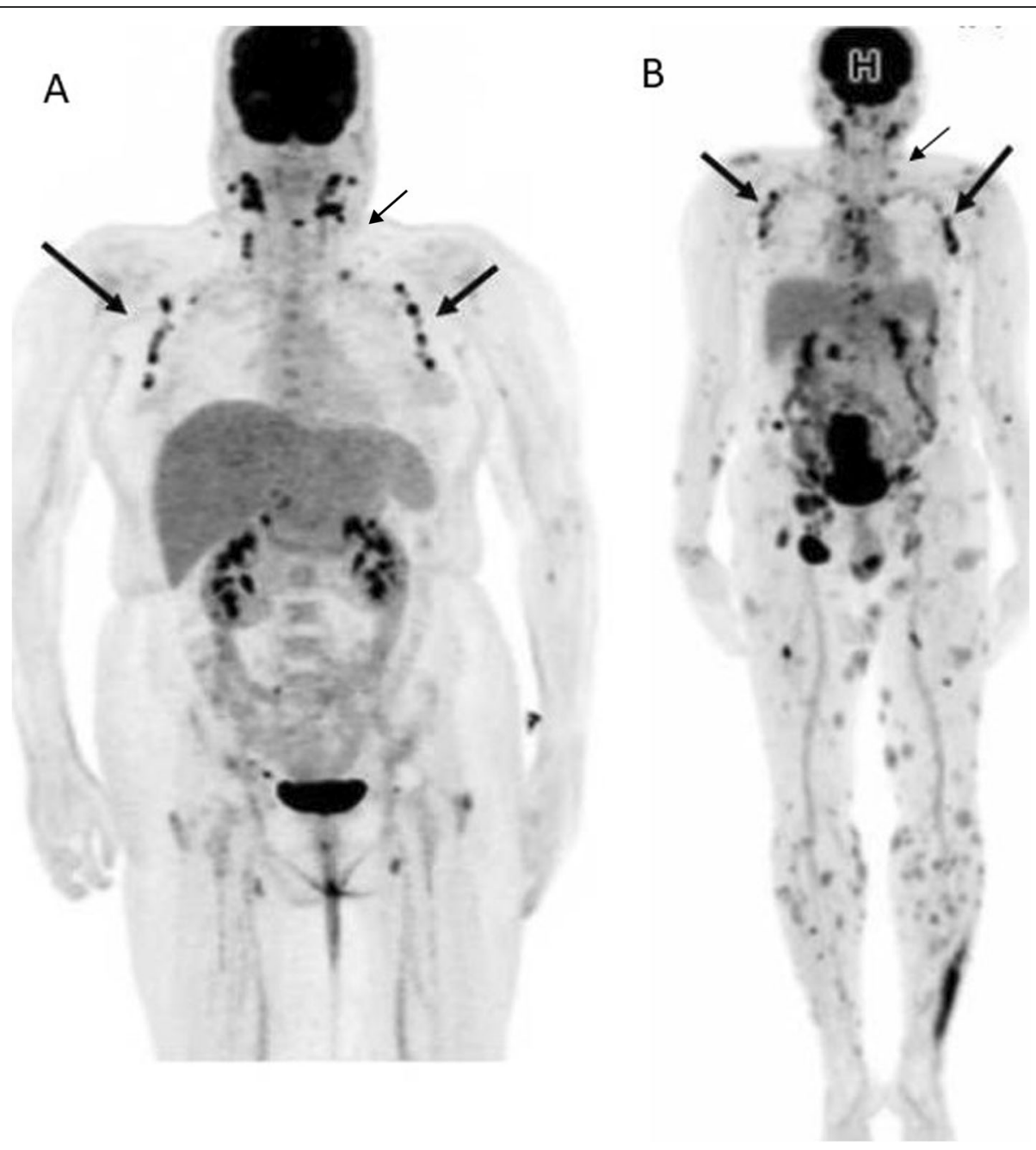

Fig. 1 a Whole body FDG PET (MIP, maximum intensity projection image, head to mid-thigh) image of a 48-year-old lady with headache and PUO. The image shows FDG avid "string of pearls sign" due to extensive supradiaphragmatic nodal lesions (arrows depict bilateral cervical, supraclavicular, pectoral, and axillary nodes) in a case of Kikuchi disease with associated meningitis. Based on PETMR, nodal biopsy, and CSF findings, the patient was appropriately managed. b FDG PET (MIP image) findings in a 61-year-old male with intermittent low-grade fever, associated generalized itching, nodular swellings in extremities, and raised inflammatory markers. Whole body FDG PETCT from head to toe was performed. The images revealed FDG avid nodal arrangement ["string of pearls sign"] in the neck and chest due to bilateral cervical, axillary, pectoral nodal involvement. There were extensive FDG avid supra and infradiaphragmatic nodal lesions along with FDG extensive cutaneous and subcutaneous nodular lesions scattered all over the body including the extremities. Mantle cell lymphoma was histologically confirmed, and the patient was subjected to chemotherapy. Thus, "string of pearls sign" is a non-specific PET finding and can be present in nodal disease of diverse etiologies

swellings in extremities for past 2 days was referred for whole body 18 F FDG PETCT. Hemoglobin was $10 \mathrm{~g} \%$ with pancytopenia at presentation. Inflammatory markers were raised. Chest X-ray was normal. No other positive symptoms or signs were elicited clinically. Whole body FDG PETCT was performed from head to toe (Fig. 1B). There were extensive FDG avid supra and infradiaphragmatic nodal lesions along with extensive FDG avid cutaneous and subcutaneous nodular lesions scattered all over the body including the extremities (Fig. 3). Based on FDG PET findings, biopsy was attempted from the right axillary nodes; immunohistochemistry revealed mantle cell lymphoma. The final diagnosis was MCL with widespread exceedingly rare extranodal lymphomatous involvement of cutaneous and subcutaneous nodules. The patient was subjected to systemic chemotherapy with tyrosine kinase inhibitor, i.e., ibrutinib. The patient is on the 2nd month of therapy and is doing well clinically.

\section{Discussion}

KFD was first described in 1972 as a lymphadenitis with specific histopathologic findings, generally self-limiting disease affecting predominantly Asian females [5]. Cerebral and pulmonary involvement is extremely rare. 


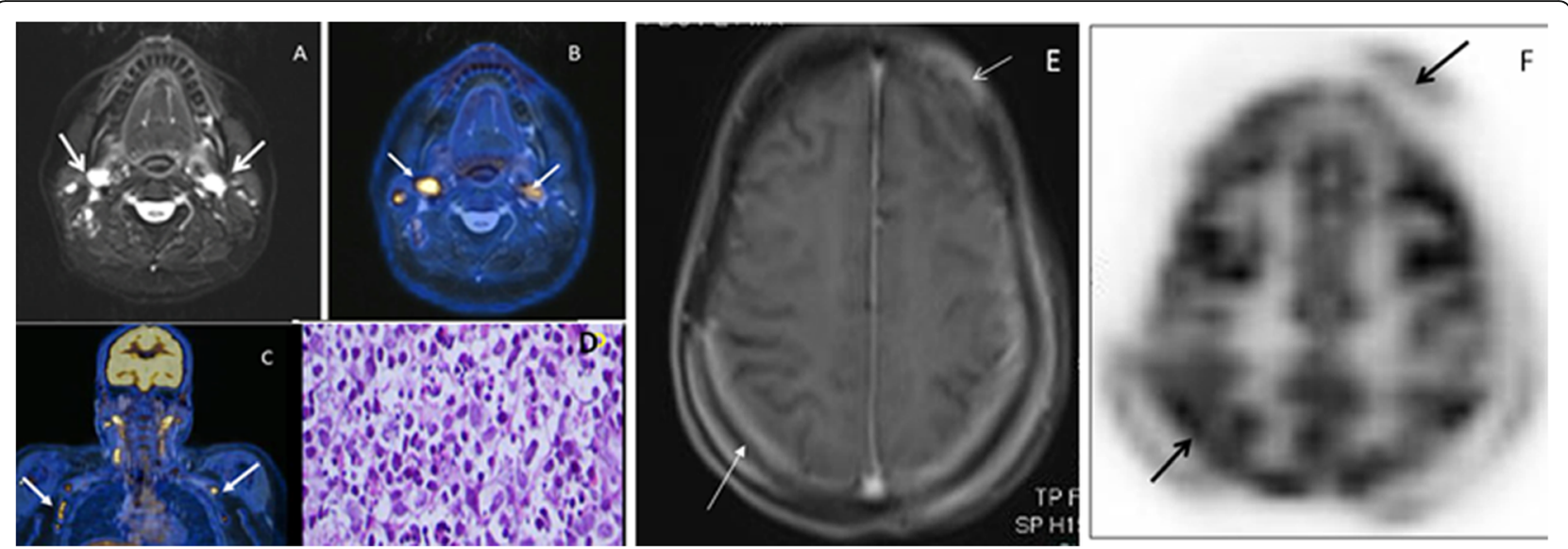

Fig. 2 a T2-weighted STIR image of the neck (transaxial) showing bilateral level 2 lymph nodes in patient with KFD. b Fused FDG PET and T2weighted STIR image (transaxial) showing FDG avid bilateral level 2 nodal lesions. c Fused FDG PET MR (coronal) image of the head, neck, and thorax showing "string of pearls sign." The arrows depict the array of FDG avid lymph nodal lesions on both sides of neck and thorax (cervical, supraclavicular, axillary, and deep pectoral stations). $\mathbf{d}$ Biopsy sections from the left axillary lymph node show partly preserved architecture with reactive follicles in the cortex. Intervention seen in the sheets of histiocytes along with admixed necrotic areas with nuclear debris. The cells have moderate pale/vacuolated cytoplasm, round to oval nuclei with open chromatin, and small prominent nucleoli. Few cells appear crescentic. No evidence of malignancy. Lymph node biopsy indicates necrotizing lymphadenitis from KFD due to the histiocytic cellular infiltrate along with phagocytic debris and crescent nuclei. e MR brain transaxial image shows diffuse enhancement and thickening of pachymeninges (arrows), suggestive of meningitis. f Fused PETMR brain transaxial image (arrows) depicts sites of abnormal increased FDG uptake corresponding to MR detected meningeal enhancements (pachymeningitis)

Anatomic and functional imaging procedures like CT and PET are not diagnostic for KFD. The affected lymph nodes in KFD appear enlarged, lose their normal shape, and acquire an abnormal round shape with associated FDG avidity indicating infection, inflammation, or a neoplastic underlying pathology [6]. The nodes may show unilateral or bilateral involvement. Reports show KFD's predilection for cervical lymphadenopathy, small- or medium-sized similar to our case. Nodal involvement is usually discrete and involves levels II, III, and IV with CT findings of low attenuation nodes [6]. In our study, we found bilateral involvement of discrete supradiaphragmatic (cervical, axillary, and deep pectoral) along with fewer infra diaphragmatic (periportal, portocaval and inguinal) nodal involvement. None of the involved nodes displayed matted appearance. Nodal size ranged from 10 to $38 \mathrm{~mm}$ with SUV max range of 3 to $6.4 \mathrm{~g} / \mathrm{ml}$. One of the studies reported extensive discrete and matted lymph nodes [7]. Gold standard for the diagnosis of KFD is biopsy of the affected lymph node [6]. Treatment is supportive with nonsteroidal anti-inflammatory drugs and corticosteroids. Recurrent aseptic meningitis in association with KFD is extremely rare as in our case and remains a diagnostic challenge. Acute cerebellar ataxia, acute brachial neuritis, and brainstem encephalitis are other rare CNS complications associated with KFD [8].

Mantle cell lymphoma (MCL) is a type of aggressive non-Hodgkin's lymphoma (NHL) occurring in the elderly, comprising about $6 \%$ of NHL cases [9] with poor prognosis. Skin involvement in MCL is exceedingly rare, found in $2-6 \%$ of patients [9]. MCL is a subtype of B cell lymphoma, due to $\mathrm{CD} 5$ positive antigen-naive pregerminal center B cell within the mantle zone that surrounds normal germinal center follicles. MCL cells generally overexpress cyclin D1 due to a $\mathrm{t}(11: 14)$ chromosomal translocation in the DNA [10]. There is not much data on the role of FDG PET in MCL; however, as in other lymphoma subtypes, it has been found to be useful in staging and therapy response evaluation. In the LyMaPET project (a prospective, multicenter, international, randomized phase III trial), PET findings were reviewed in young previously untreated MCL patients at baseline and after undergoing rituximab maintenance therapy post autologous stem-cell transplantation (ASCT). Two hundred ninety nine patients were enrolled in the LyMa study; FDG PET data from 104 patients were retrieved from 28 different centers. Patients with a high SUV max (>10.3 g/ml) had a shorter progression free survival at 4 years. The trial results supported the existence of a close relationship between tumor cell biology and SUV in MCL as with other lymphomas. When compared to Ki67 positivity in a tumor biopsy, FDG PET had the advantage of being a whole-body non-invasive technique [11]. MCL with extranodal lymphomatous involvement (spleen, liver, bone marrow, skin) was found to be a poor prognostic indicator signifying advanced disease (stage IV) and calls for aggressive management in the form of systemic chemotherapy regimens. 

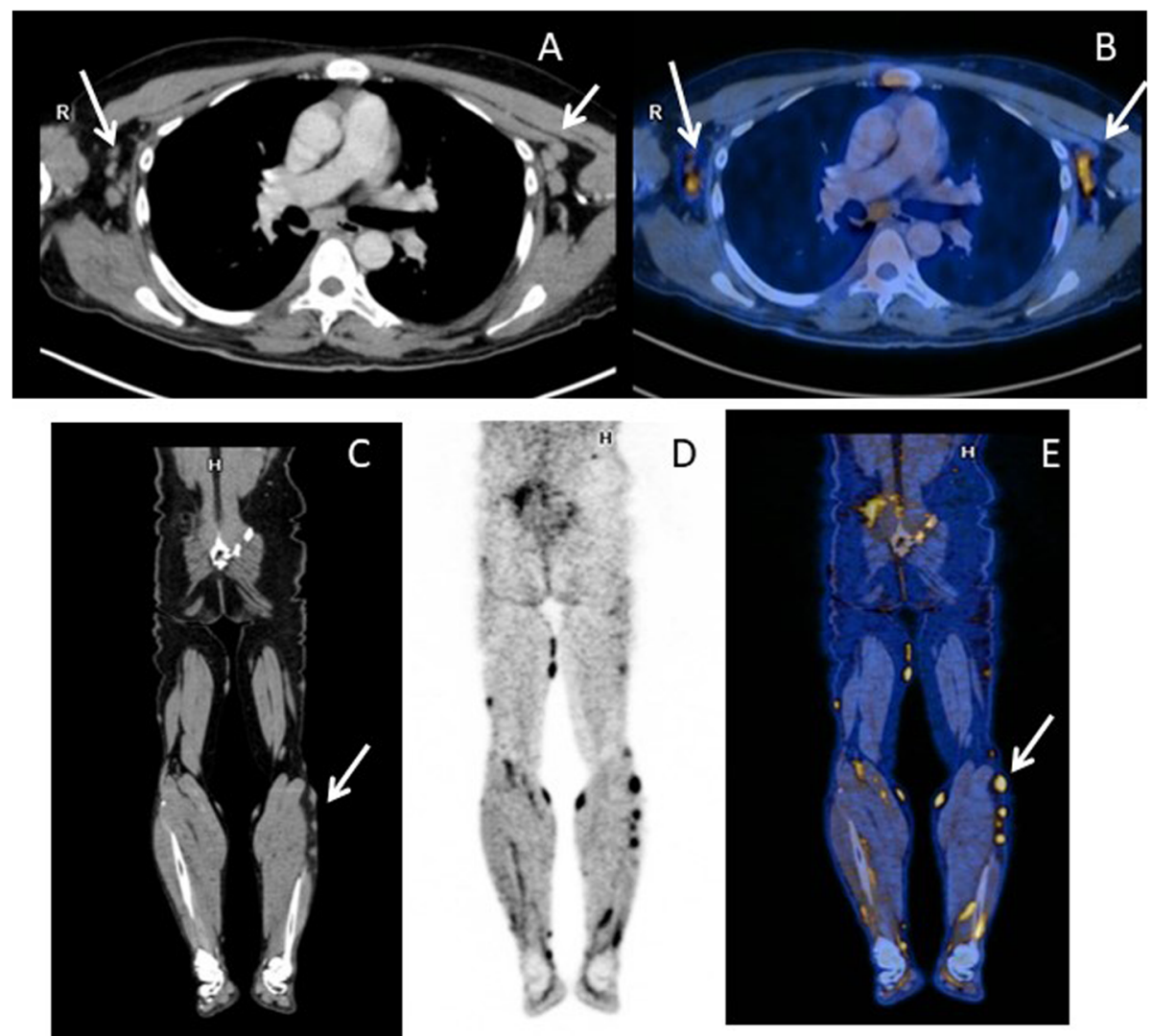

$\mathrm{D}$

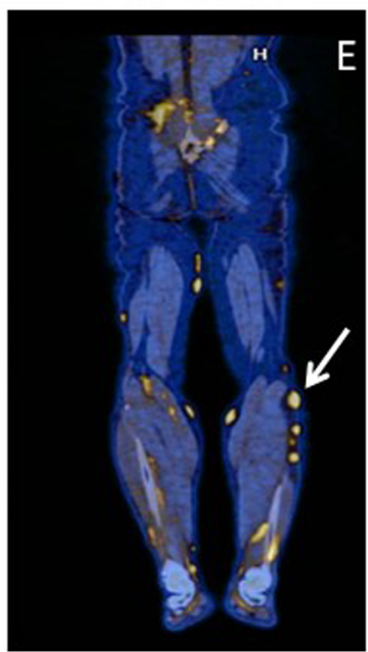

Fig. 3 The case of mantle cell lymphoma with "string of pearls" sign on PET depicting nodal involvement. PETCT images (a contrast-enhanced CT chest, $\mathbf{b}$ fused PETCT transaxial image, $\mathbf{c}$ contrast-enhanced $C T$ image in coronal section of lower limbs, $\mathbf{d}$ fused PETCT lower limb image in coronal view) show FDG avid bilateral axillary lymphadenopathy (arrows) along with extensive FDG avid nodular subcutaneous deposits in extremities (arrow)

\section{Conclusion}

FDG PET has helped in identifying the cause of febrile illness in both patients using a single imaging modality. It has been instrumental in the identification of exceedingly rare associations in both patients which has further helped in adding new line of therapy and optimizing patient management. FDG PET should be recommended as a procedure of choice in PUO when pathology still remains elusive after thorough clinical and biochemical panel of investigations. FDG avid nodal disease is a non-specific finding but identifies the accurate site for biopsy that helps in clinching the diagnosis. The node displaying the highest SUV max with easy approachability for biopsy is found to have the highest diagnostic yield that can lead to the right management.

\section{Abbreviations}

PUO: Pyrexia of unknown origin; ${ }^{18}$ F FDG PET: ${ }^{18}$ Fluorine fluorodeoxyglucose positron emission tomography; ESR: Erythrocyte sedimentation rate; CSF: Cerebrospinal fluid; KFD: Kikuchi Fujimoto disease; MCL: Mantle cell lymphoma; PETMR: Positron emission tomography-magnetic resonance imaging; GLUT: Glucose uptake transport; NHL: Non-Hodgkin's lymphoma

\section{Acknowledgements}

None.

\section{Authors' contributions}

SS, SP, and SY conceived the idea and contributed to the workup and draft of the manuscript. SS and SP contributed to the proofreading and finalization of the manuscript; SY provided the patient-related information; SS and SP interpreted the imaging findings as well as their interpretation. All authors have read and approved the manuscript.

\section{Funding}

No funding sources or grants.

Availability of data and materials Not applicable.

\section{Declarations}

Ethics approval and consent to participate

PET is an established routine imaging procedure; ethics approval is not needed. Both patients have given their consent for imaging.

\section{Consent for publication}

Both patients have given their written consent for publication. This was obtained directly from patient as they are adults (legal age).

\section{Competing interests}

The authors declare that they have no competing interests.

\section{Author details}

${ }^{1}$ Amrita School of Medicine, Amrita Vishwa Vidyapeetham, Ponekkara Post, Cochin, Kerala 682041, India. ${ }^{2}$ Department of Nuclear Medicine \& Molecular 
Imaging, Amrita Institute of Medical Sciences, Ponekkara Post, Cochin, Kerala 682041, India.

Received: 4 November 2020 Accepted: 7 March 2021

Published online: 30 March 2021

\section{References}

1. Petersdorf RG, Beeson PB (1961) Fever of unexplained origin: report on 100 cases. Medicine 40:1-30

2. Zhu W, Cao W, Zheng X, Li X, Li Y, Chen B, Zhang J (2020) The diagnostic value of $18 \mathrm{~F} \mathrm{FDG} \mathrm{PET/CT} \mathrm{in} \mathrm{identifying} \mathrm{the} \mathrm{causes} \mathrm{of} \mathrm{fever} \mathrm{of} \mathrm{unknown}$ origin. Clin Med 20(5):449-453. https://doi.org/10.7861/clinmed.2020-0268

3. Fernandez C, Beeching NJ (2018) Pyrexia of unknown origin. Clin Med (Lond) 18(2):170-174. https://doi.org/10.7861/clinmedicine.18-2-170

4. Arnon-Sheleg E, Israel O, Keidar Z (2020) PET/CT imaging in soft tissue infection and inflammation-an update. Semin Nucl Med 50(1):35-49. https://doi.org/10.1053/j.semnuclmed.2019.07.005

5. Kikuchi M (1972) Lymphadenitis showing focal reticulum cell hyperplasia with nuclear debris and phagocytes. Acta Hematol Jpn 35:379-380

6. Mghanga FP, Bakari KH, Mayala HA (2017) The role of PET/CT in the diagnosis of Kikuchi-Fujimoto disease: a case series and review of the literature. J Mol Imaging Dynamic 7(2):139. https://doi.org/10.4172/2155-993 7.1000139

7. Kong E, Chun K, Hong Y, Hah J, Cho I (2013) 18F-FDG PET/CT findings in patients with Kikuchi disease. Nuklearmedizin. 52(3):101-106. https://doi. org/10.3413/Nukmed-0513-12-06

8. Yelsangikar KR, Srikanteswara P-k (2014) Kikuchi-Fujimoto's disease presenting as acute cerebellar ataxia. J Pediatr Neurol 12:221-224

9. Swerdlow SH, Campo E, Pileri SA, Harris NL, Stein H, Siebert R, Advani R, Ghielmini M, Salles GA, Zelenetz AD, Jaffe ES (2016) The 2016 revision of the World Health Organization classification of lymphoid neoplasms. Blood. 127(20):2375-2390. https://doi.org/10.1182/blood-2016-01-643569

10. Golardi N, Velasco MR, Elghetany MT (2009) Marginal zone variant of mantle cell lymphoma: CD5-negative cyclin D1-positive variant posing a diagnostic dilemma. Pathol Int. 59(5):317-321. https://doi.org/10.1111/j.1440-1827.2009. 02372

11. Bailly C, Carlier T, Berriolo-Riedinger A, Casasnovas O, Gyan E, Meignan M, Moreau A, Burroni B, Djaileb L, Gressin R, Devillers A, Lamy T, Thieblemont C, Hermine O, Kraeber-Bodéré F, le Gouill S, Bodet-Milin C (2020) Prognostic value of FDG-PET in patients with mantle cell lymphoma: results from the LyMa-PET Project. Haematologica 105(1):e33-e36. https://doi.org/10.3324/ha ematol.2019.223016

\section{Publisher's Note}

Springer Nature remains neutral with regard to jurisdictional claims in published maps and institutional affiliations.

\section{Submit your manuscript to a SpringerOpen ${ }^{\circ}$ journal and benefit from:}

- Convenient online submission

- Rigorous peer review

- Open access: articles freely available online

- High visibility within the field

- Retaining the copyright to your article

Submit your next manuscript at $\boldsymbol{\nabla}$ springeropen.com 